\title{
Laboratory testbed for the calibration and the validation of the shadow position sensor subsystem of the PROBA3 ESA mission
}

Davide Loreggia, Luca Zangrilli, Gerardo Capobianco, Giuseppe Massone, Massimiliano Belluso, et al.

Davide Loreggia, Luca Zangrilli, Gerardo Capobianco, Giuseppe Massone, Massimiliano Belluso, Silvano Fineschi, Francesco Amadori, Vladimiro Noce, Alessandro Bemporad, Marta Casti, Gianalfredo Nicolini, Federico Landini, Maurizio Pancrazzi, Marco Romoli, "Laboratory testbed for the calibration and the validation of the shadow position sensor subsystem of the PROBA3 ESA mission," Proc. SPIE 11852, International Conference on Space Optics ICSO 2020, 118526Q (11 June 2021); doi: 10.1117/12.2600312

SPIE Event: International Conference on Space Optics - ICSO 2021, 2021, Online Only 


\section{International Conference on Space Optics-ICSO 2020}

Virtual Conference

30 March-2 April 2021

Edited by Bruno Cugny, Zoran Sodnik, and Nikos Karafolas
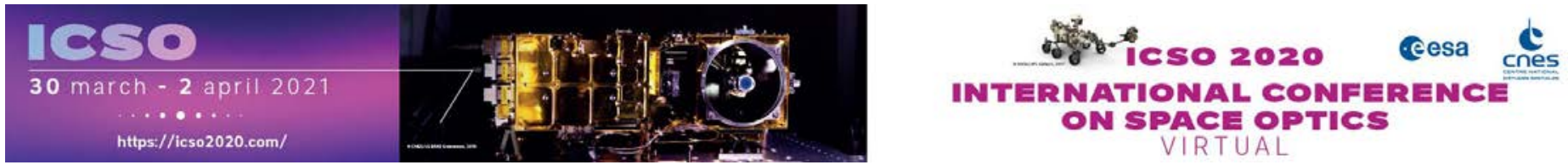

\section{Laboratory testbed for the calibration and the validation of the shadow position sensor subsystem of the PROBA3 ESA mission}

\section{Cesa issopreasings denes}




\title{
Laboratory testbed for the calibration and the validation of the Shadow Position Sensor subsystem of the PROBA3 ESA mission
}

Davide Loreggia $^{a}$, Luca Zangrillia ${ }^{a}$, Gerardo Capobianco ${ }^{a}$, Giuseppe Massone ${ }^{a}$, Massimiliano Belluso $^{\mathrm{b}}$, Silvano Fineschi ${ }^{\mathrm{a}}$, Francesco Amadori ${ }^{\mathrm{a}}$, Vladimiro Noce ${ }^{\mathrm{c}}$, Alessandro Bemporad ${ }^{\mathrm{a}}$, Marta Casti ${ }^{\mathrm{a}, \mathrm{e}}$, Gianalfredo Nicolini ${ }^{\mathrm{a}}$, Federico Landini ${ }^{\mathrm{a}}$, Maurizio Pancrazzi ${ }^{\mathrm{a}}$, and Marco Romoli $^{\mathrm{d}}$

${ }^{a}$ INAF - Astrophysical Observatory of Turin, Via Osservatorio, 20, I-10025 Pino Torinese, Torino, Italy

${ }^{b}$ INAF - Astrophysical Observatory of Catania, Via S. Sofia, 78, I-95123, Catania, Italy ${ }^{\mathrm{c}}$ INAF - Astrophysical Observatory of Arcetri, Largo Enrico Fermi, 5, 50125 Firenze, Italy

${ }^{\mathrm{d} U n i v e r s i t y ~ o f ~ F l o r e n c e, ~ D e p . ~ o f ~ P h y s i c s ~ a n d ~ A s t r o n o m y, ~ V i a ~ G . ~ S a n s o n e, ~ 1, ~ I-50019 ~ S e s t o ~}$ Fiorentino, Florence, Italy

eALTEC S.p.A., Corso Marche, 79, 10146 Torino, Italy

\begin{abstract}
The PROBA3 mission of the European Space Agency is the first formation flying (FF) mission that will be flown in high elliptic geocentric orbit aiming at verifying and validating different metrology control systems and algorithms in order to realize and maintain the formation of two independent spacecraft, in total autonomy. The final target accuracy for the relative and absolute alignment of the two satellites is of about $2 \mathrm{~mm}$ over an inter satellite distance of $144.3 \mathrm{~m}$. During the FF, the two spacecraft will realize a giant coronagraph with the external occulter on one payload and the telescope on the other one. The Sun Corona observation will be the scientific tool for the FF validation. Between the different metrology systems that will be tested, the Shadow Position Sensor (SPS) is the most challenging one, aiming at returning the relative and absolute position of the formation with the finest accuracy: $0.5 \mathrm{~mm}$ out of the guidance and navigation and control loop and $2 \mathrm{~mm}$ within the loop. The mission program is now in the Phase $\mathrm{D}$ with the realization and the testing of the flight model. Due to the high expected performance, a fine calibration of the SPS subsystem is mandatory. In this paper, we discuss the radiometric and spectral calibration plan, the algorithm validation procedure, and the laboratory test-bed realized to reproduce the in-flight observation conditions of the SPS by using a set of calibrated LED and a mechanical set-up equivalent to the SPS system. Preliminary results are also reviewed.
\end{abstract}

Keywords: Formation Flying, Positioning Sensor, Space Mission, Coronagraph, Metrology.

\section{INTRODUCTION}

Formation flying of multiple satellites is a mission concept that has been on the path for challenging space projects from the last decades of the XX century [1],[2]. In particular, the great advantages rising from astronomical FF mission gathered an increasing interest within the scientific community, becoming one of the main drivers for the study, design, and planning of next generation space missions. Reaching the goal unavoidably asks for thinking and developing technological demonstrators in order to test, verify, and validate different concepts for the metrology control system, the core for realizing the FF. The success of the PRISMA mission [3], even accounting of its intrinsic limitations due to the lack of fine actuation and adequate optical metrology, and the unfavourable gravitational environment of the low elliptical orbit, demonstrated that the FF is a mature technology. To step forward, in recent years, the most effort was focused on the accurate modelling of high accuracy metrology and on the development of control pipelines that permit the automatizing of very fine control loops yielding to fly

Reference contact: Davide Loreggia, E-mail: davide.loreggia@inaf.it 
multiple spacecraft formation in more favourable gravitational environments and in complete autonomy.

The PROBA3 (PRoject for On-Board Autonomy) mission of the European Space Agency [4], represents a cornerstone project aimed at reaching these objectives. PROBA3 is a two-spacecraft formation flying on a High Elliptical Orbit (HEO) with perigee at $600 \mathrm{Km}$ and apogee at $60000 \mathrm{Km}$, in a full automatic way. Several metrology hardware and software systems have been designed for being in-flight tested and validated to confirm the FF. The PROBA3 program is now in its Phase-D stage with most of the flight models of the system and subsystems being manufactured and accepted. The assembly of the flight instrument on the platform is planned for the next year (2022) with the launch currently scheduled for beginning of 2023.

\section{PROBA3 MISSION AND THE SHADOW POSITION SENSORS (SPS)}

The two spacecraft composing the PROBA3 mission will acquire the formation during the transit at the apogee, taking advantage of the minimum gravity gradient. During the FF phase, they will realize a giant externally occulted coronagraph, as a rigid structure. The Coronagraph SpaceCraft (CSC) will host the optical assembly of the coronagraph and the Occulter SpaceCraft the external occulter (EO) disk, positioned at an inter-satellite distance (ISD) of $144 \mathrm{~m}$. The successful observation of the Sun corona is the scientific target that will confirm the FF capabilities. The large occultation geometry and the occulter design will minimize the diffraction [5] so that ASPIICS is expected to return high spatial and temporal resolution images of the inner part of the solar corona, very close to the solar limb. PROBA3 will observe in visible broad and narrow band, and in polarized light from $1.08 R_{\text {Sun }}$ up to about $3 R_{\text {Sun }}$.

In order to achieve the formation-flying objectives, a suite of metrology systems will provide continuous measurements of the satellites relative and absolute position down to sub-millimetre precision [6]. Among these, the Shadows Position Sensors (SPS) is expected to return the position measurement with the highest accuracy [7]. The SPS unit consists of a mechanical flange mounted in front of the ASPIICS telescope boom, hosting an electronic board where a set of eight silicon photo-multipliers ( $\mathrm{SiPMs}$ ), positioned along a circumference concentric with the telescope entrance pupil, will measure the solar penumbra irradiance as projected by the external occulter. The ASPIICS's pupil has a radius of $25 \mathrm{~mm}$ while the SPS sensors are positioned at $55 \mathrm{~mm}$ from the centre and receive the solar radiation though a set of pinholes realized on the front surface of the mounting flange, that are properly sized to equalize the flux over each diode. The electronic boards and the mounting mechanical flange are shown in the following figure 1. When the formation will approach its nominal alignment,
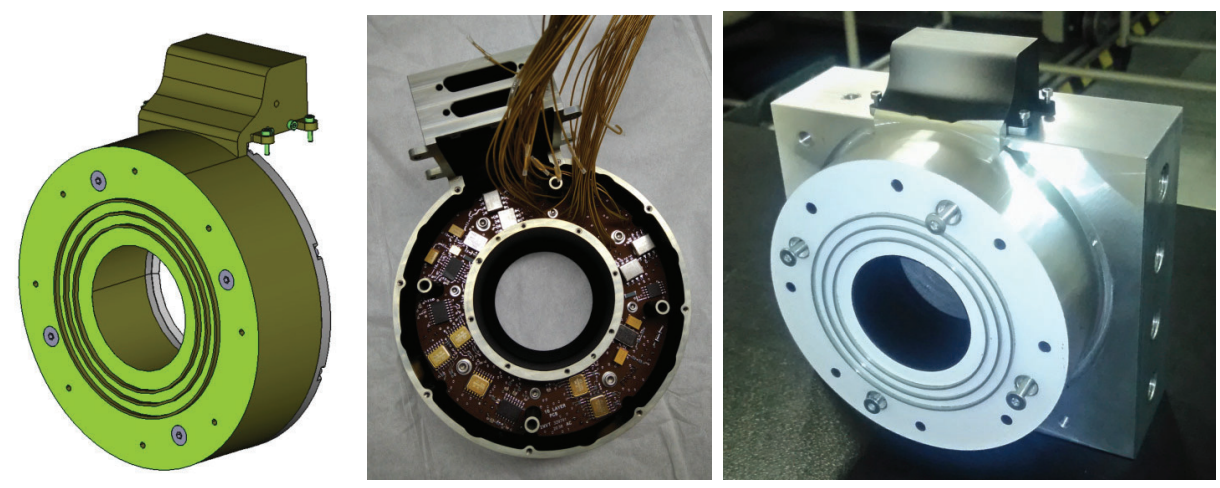

Figure 1. Left) SPS CAD rendering; center) Assembly of the manufactured SPS flange with the electronic board; right) SPS installed on the fixture for vibration qualification (same fixation interface as with the ASPIICS telescope).

with the telescope's door open, the SPS flange will be exposed to the penumbra generated by the occulter and the solar radiation distribution will be measured monitoring its symmetry with respect to the telescope line of sight. The eight SiPMs are grouped in two sets that are used separately: the set A (nominal), composed by SiPMs 1, 3, 5, 7, and the set B (redundant), composed by SiPMs 2, 4, 6, 8, as shown in figure 2 where the distribution of the SiPMs with respect to the pupil, as mounted on the telescope and respect to the penumbra, is shown. During standard operations the set A (nominal) will be operated while the set B (redundant) will be used only for calibration, or in case the nominal one will go failed [7]. Moreover, due to the need for a precise 

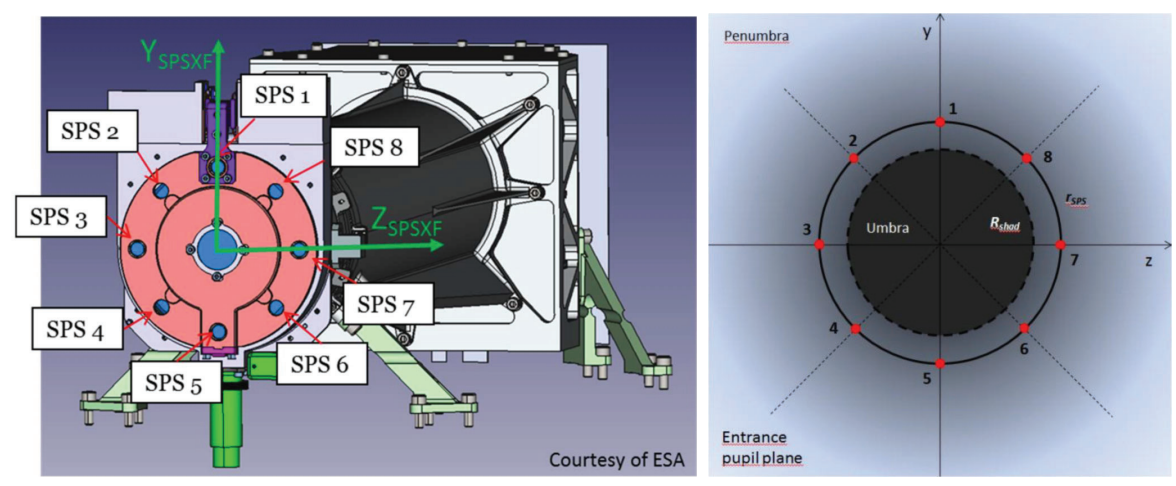

Figure 2. Left) SPS CAD rendering of the SPS as mounted on the front of the ASPIICS telescope; right) Rendering of the expected umbra and penumbra distribution on the ASPIICS's pupil plane.

in-flight calibration, the full Sun will be used as reference source and the nominal SPS set will be permitted to observe directly the Sun through a set of neutral density filters mounted in front of each diode on the front door when closed. The redundant set, on the other hand, will have clear holes in front of the diodes to permit a cross calibration during partial eclipses [8]. The SPS will observe within the waveband [500-660]nm that has been selected to reduce the impact of the temperature dependence of the SiPMs responsivity. The Sun light is filtered by applying a band-pass filter coating on the entrance windows of the SiPM caps. The SPS system is passively powered by the Coronagraph Control Box of the ASPIICS instrument, that commands the two sets separately. The FF alignment measurement accuracy required to the SPS is $500 \mu m(3 \sigma)$ for lateral movements out of the control loop, $2 \mathrm{~mm}$ within the control loop, and $50 \mathrm{~mm}(3 \sigma)$ for longitudinal movements, within a $3 \mathrm{D} F F$ requirement box of $(20 \times 20 \times 200) \mathrm{mm}^{3}$. Besides, the SPS should be able to return a position estimation on a wider 3D goal box of $(200 \times 200 \times 2000) \mathrm{mm}^{3}$ without any accuracy specification [7]. The SiPMs generate a current proportional to the received irradiance. The currents are then converted in voltage by the proximity electronics and amplified by a 2-stage amplification chain [9]. Finally, the signals are digitized by 12-bit ADCs. The double gain processing, high gain (HG) ad low gain (LG), is needed to cover the very large dynamic range of the expected illumination (from umbra to full Sun illumination) and to satisfy the accuracy requirements that demands for a high sensitivity to very small flux variations.

The digital readouts are transferred to the on-board software, where a dedicated metrology algorithm processes the SPS measurements to calculate the FF alignment [10] by comparing the readouts differences between opposite diodes. To convert the measured irradiances into the displacement of the coronagraph with respect to the umbra, the algorithm requires an accurate knowledge of the light distribution on the SPS plane. This is obtained by a detailed mathematical modelling of the penumbra profile [11] and by a proper in-flight calibration of the actual Sun light distribution [8]. On the other hand, on-ground calibration plays the critical role to describe the expected response of the SPS diodes, yielding to their complete characterization. In this paper, we describe the test-bed that has been designed, manufactured, and set-up at the Astrophysical Observatory of Turin, one of the branch of the Italian National Institute for Astrophysics, to perform the SPS sub-system calibrations. The test procedure and the preliminary results obtained are also reviewed.

\section{SPS ON-GROUND CALIBRATION TEST}

\subsection{Test objective}

The objective of the on-ground calibration of the SPS subsystem is to obtain the most detailed description of the radiometric and spectral behaviour of the 8 SPS sensors, in order to provide the algorithm with the required coefficients needed to properly manage the SPS measurements. This is mandatory to account for all the differential contributions between the couples of SiPMs used for the penumbra monitoring that are critical for the full acquisition chain. The on-ground calibrations of the SPS is a multi-step activity that includes a preliminary verification using an Advanced Demonstration Model (ADM), the test of the SPS flight model (FM) and, eventually, the calibration of the SPS units when assembled on the ASPIICS telescope, before being integrated on 
the spacecraft platform. At the moment of the presentation of this paper, the preliminary verification with the ADM is being completed. The other steps, and the final results obtained on the flight unit will be subject of future publications.

The ADM of the SPS consists of a Printed Circuit Board (PCB) functionally equivalent to FM from which it differs only for the presence of commercial components instead of space qualified ones. The ADM was manufactured basing on the SPS PCB design with the aim to perform testing with a more comfortable attitude. The ADM model (as well the SPS-EQM that did undergo a successful qualification campaign in 2019), and the SPS-FM, that is now entering the acceptance campaign, is equipped with an FPGA-based interface board and a PC program simulating the on-board software and its readout procedures. In figure 3, the ADM before being prepared for mounting on the mechanical interface used for the calibration tests, and the control GUI are shown..
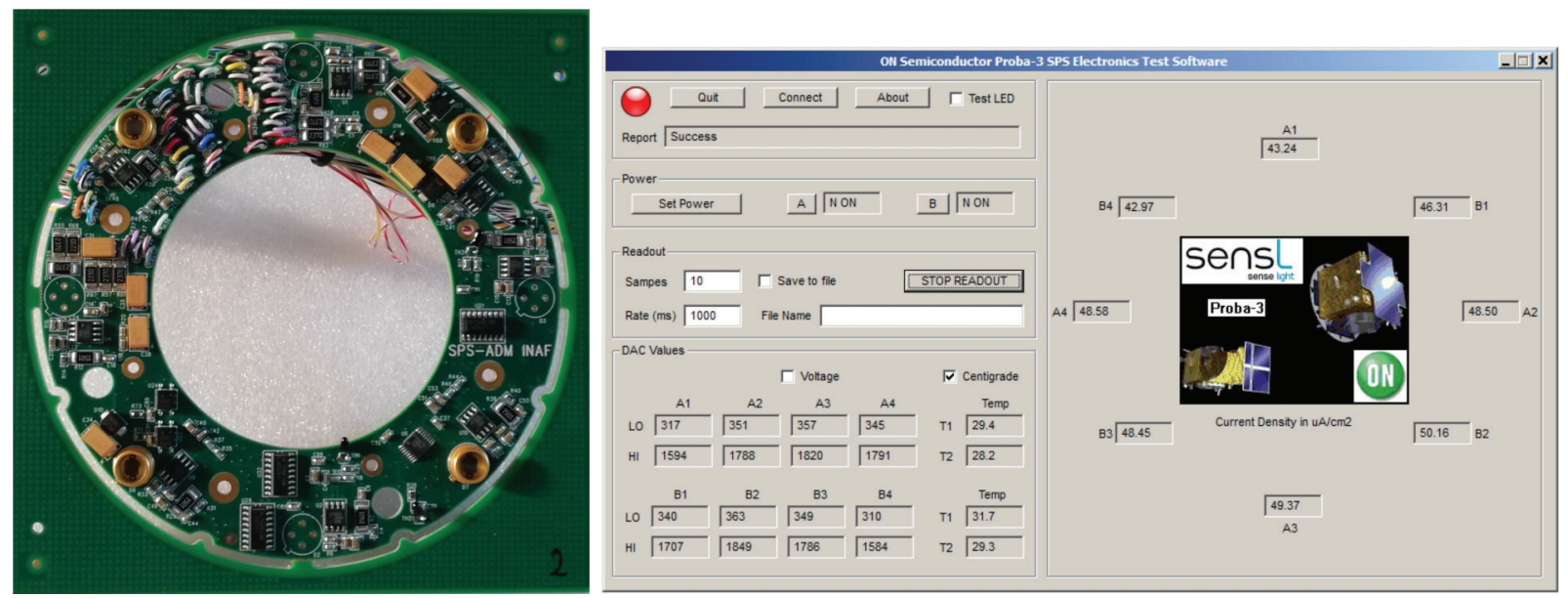

Figure 3. Advanced Demonstration Model of the SPS (the ADM with only 4 SiPMS mounted is shown in order to highlight the mounting electronics geometry).

Several tests have been identified in order to cover the different aspects of the SPS calibration, which are summarized in table 1, also indicating if the test is applicable to the ADM and/or to the FM. The full characterization

Table 1. Calibration test summary.

\begin{tabular}{|c|c|c|}
\hline Measurement Type & ADM & FM \\
\hline Time stability test & $\mathrm{X}$ & \\
\hline Radiometric calibration (at room temperature) & $\mathrm{X}$ & $\mathrm{X}$ \\
\hline Temperature dependence of the radiometric calibration & $\mathrm{X}$ & \\
\hline Spectral dependence of the radiometric calibration & $\mathrm{X}$ & \\
\hline Error budget verification & $\mathrm{X}$ & \\
\hline Algorithm verification & $\mathrm{X}$ & $\mathrm{X}$ \\
\hline
\end{tabular}

is being done with the ADM, such as the complete verification and validation of the operational functionalities and of the calibration impacts on the overall system performances. Only the radiometric calibration at room temperature and the algorithm verification tests are planned using the FM. 


\subsection{Test description and set-up}

\subsubsection{Time stability test and radiometric calibration}

The time stability test aims at verifying how the SPS outputs vary when measuring at constant input light flux, for a long time period. These measurements are performed at three illumination conditions: in low light regime (i.e., SPS output $\approx 100 \mathrm{DNs}$, high gain); at medium light regime (i.e., SPS output $\approx 1000 \mathrm{DNs}$, high gain); at high light regime, close to the saturation of the high gain channel (i.e., SPS output $\approx 4000 \mathrm{DNs}$, high gain). The monitoring is performed for about 2 hours corresponding to $33 \%$ of the expected FF operation duration for each orbit. The results are shown in section 5.1; the temporal stability of the light source is subtracted from these measurements (see par.4).

The radiometric calibration, performed at room temperature (i.e., $24^{\circ} \mathrm{C}$ ) and at different temperature set-points (i.e., $30^{\circ} \mathrm{C}, 35^{\circ} \mathrm{C}, 41^{\circ} \mathrm{C}$ and $50{ }^{\circ} \mathrm{C}$ ), allows to estimate the overall responsivity of the SPS board, i.e., the DNs generated for each channel of the SPS per unit of input irradiance $\left[\frac{W}{\mathrm{~cm}^{2}}\right]$ (SiPM are $3 \times 3 \mathrm{~mm}^{2}$ wide). From these measurements, the parameters $k_{i}$ are evaluated. Since the SPS algorithm is based on the difference between the outputs from two opposite SiPMs [10], the $k_{i}$ parameters works as "cross-normalization" factors that permit to equalize the output of the different channels when measuring the same irradiance. The results of the test at room temperature for the ADM are reported in section 5.2.

The main requirement for realizing the calibration of the SPS system is the use of a proper light source. This is obtained by using a set of LED [4] emitting at $623 \mathrm{~nm}$ wavelength with a band-pass of $23 \mathrm{~nm}$ that have been radiometrically calibrated, as discussed in section 4. In order to cover the dynamic range of the Sun irradiance and to guarantee the required sensitivity to the expected illumination, three LEDs with different emitting characteristics are used to illuminate each SPS SiPM. The light exiting each LED is injected within an optical guide and the three light guides are merged by multi-junction interface to a single optical fibre that brings the light in front of a single SPS diode. A mechanical interface has been designed and manufactured to guarantee the proper matching between the emitting fibers and the SiPM on the ADM. This interface is built by two different aluminum-made flanges that are mounted one in front to the other and fastened together to realize a single rigid structure [12]. A first flange has been realized to mimic the mechanical flange being used in flight, even if with a simplified design. This flange has $\Phi=2.5 \mathrm{~mm}$ pinholes properly positioned in front of the SPS SiPM, as in the FM, with the same mounting interfaces in order to install the ADM electronic board as it will be done with the FM PCB.

The second flange, also named counter-flange, has 8 threaded coupling junctions where the SMA fiber connector can be screwed exactly in front of the 8 pinholes of the first flange. The two flanges are mounted with proper spacers in order to have the beam exiting the optical fiber spreading enough to make uniform the illumination over the SPS diode, and locked each other by a mechanical bridge that is also the interface link with the holder used to mount the whole test bed on the optical bench. Once the optical fibers are attached to the counter-flange, the set up results as in figure 4. 12 LEDs are used as full illumination system [4], grouped by three for each SiPM, so the current set up permits to test only one set of SPS at a time (i.e., 4 SiPMs only). The set-up discussed in this paper, will be used for testing the SPS sub-unit at system level, as well, i.e., with the SPS FM mounted in front of the FM telescope. In that case, the counter-flange with the fibre outputs will be installed directly in front of the FM flange.

\subsubsection{Spectral calibration}

Being that the FM SPS has a band-pass filter on the front windows limiting the sensitivity of the SiPMs to the waveband [500-660] nm, a specific test has been performed on the ADM (whose SiPMs have no filters) to evaluate the impact on the measurements of the $k_{i}$ factors when illuminating the SiPMs with an almost monochromatic source within the considered waveband. This test yields to access the spectral dependence of the radiometric calibration. A dedicated set-up has been used for the verification of the spectral variation of the SiPMs responsivity using a stabilized Quartz Tungsten Halogen (QTH) lamp coupled with a monochromator. The light was driven from the exit slit of the monochromator to the SPS board by using a light guide and the wavelengths used for the measurements have been set spanning the SPS operative waveband at steps of $(10 \pm 1) \mathrm{nm}$. The central peaks have been verified using a spectrometer and the results are shown in Figure 5. The $k_{i}$ parameters are retrieved at each wavelength and compared with the values obtained by illuminating the SiPMs with the test-bed. The results are reported in the paragraph 5.3. 

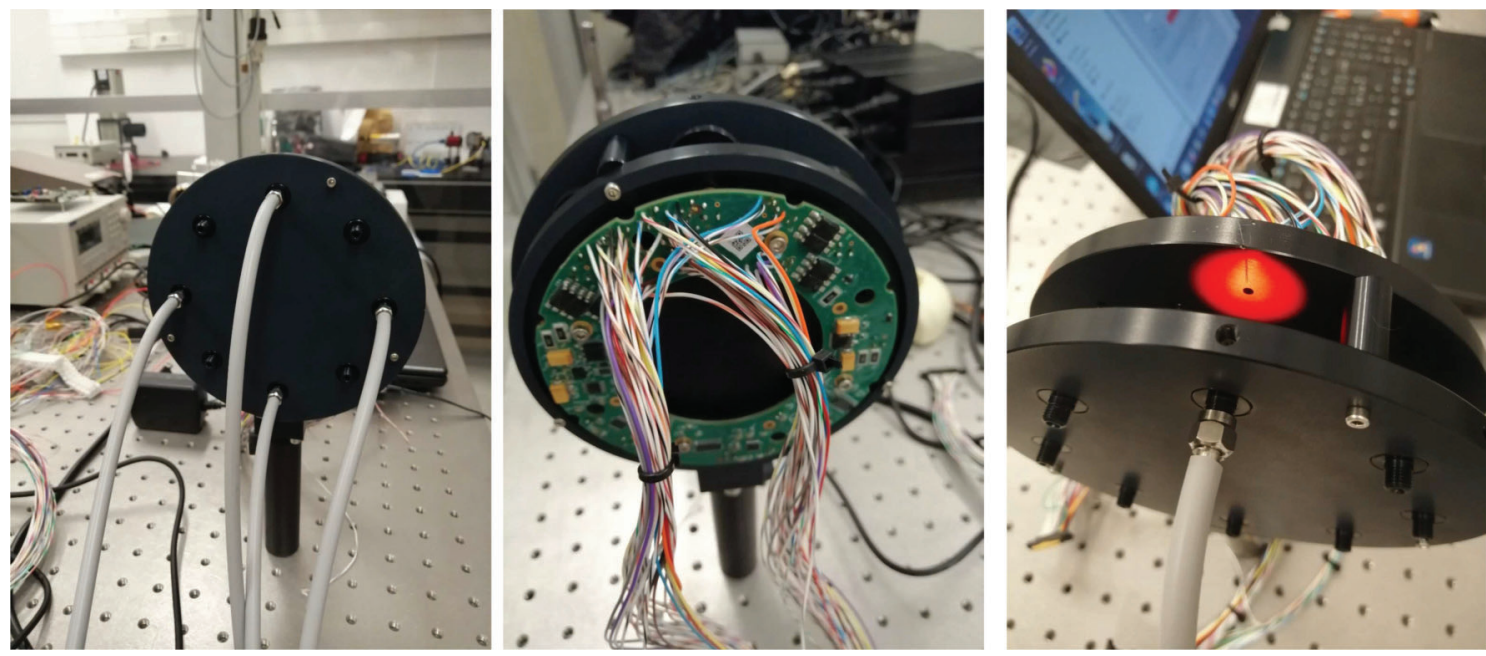

Figure 4. Calibration testbed set-up.
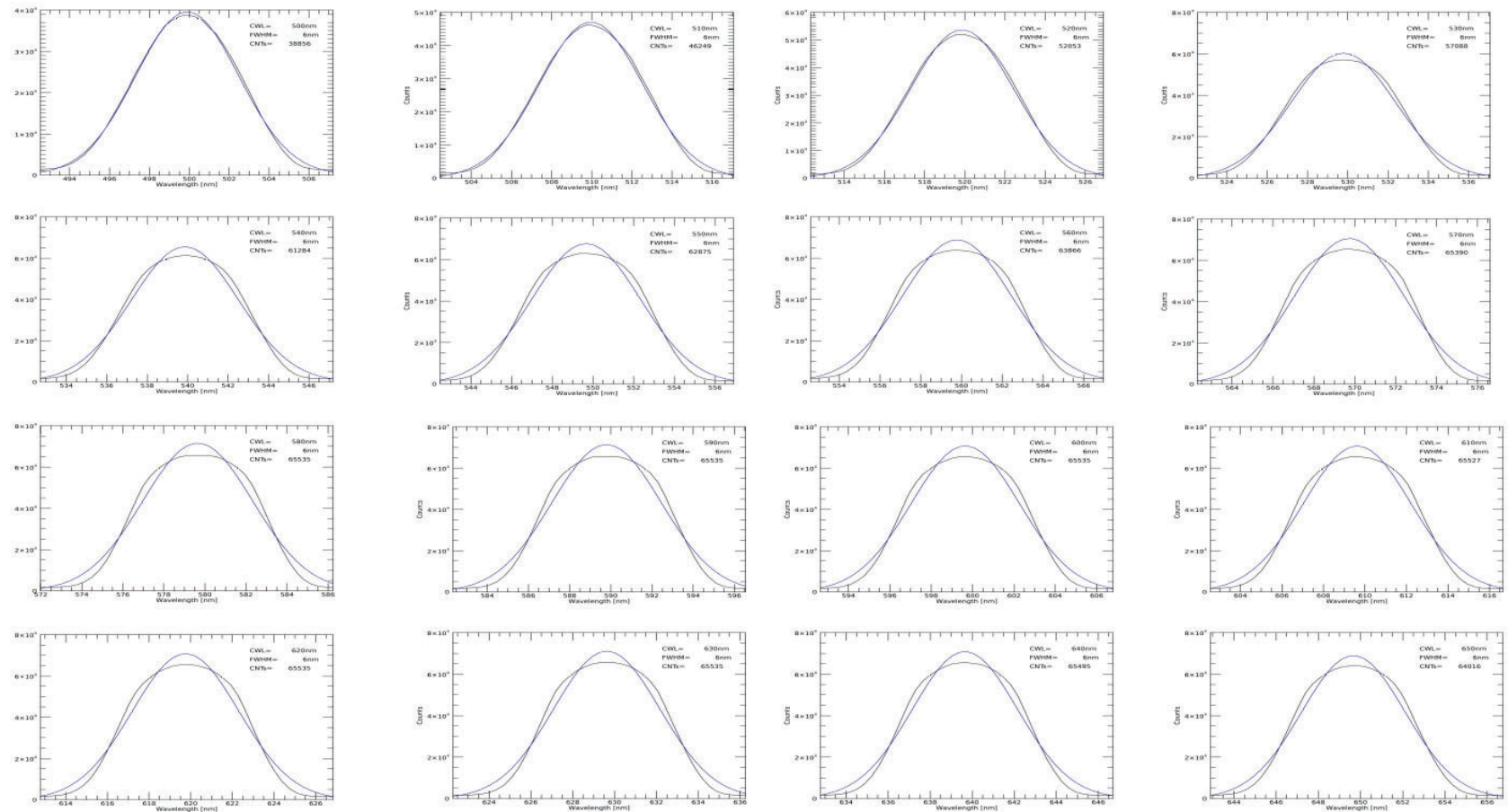

Figure 5. Monochromator wavelengths used for the spectral characterization as measured by the spectrometer. A Gaussian fit is over-plotted.

\subsubsection{Error budget verification}

In order to separately evaluate the contribution of the SiPM and of the electronics to the SPS measurement errors, we planned to perform an error budget verification test that consists in injecting the expected current generated by the SiPM when illuminated, directly into the electronic circuit bypassing the diode. This can be done by using an ADM board populated with only 4 SiPMs. The pads of the unmounted (4) SiPMs are used to input the same amount of current to the trans-impedance amplifier (TIA) from an external current generator that can be precisely tuned to reproduce the expected current output of the diodes. In this way, by comparing the digital output of the channel with the illuminated SiPM with the output from the channel externally fed, the 
contribution to the readout noise from the diode itself can be evaluated. The external current is directly injected into the PCB board using a Keithley 2634B source meter instrument. At the current level corresponding to the high gain saturation (i.e., range of $10 \mu \mathrm{A}$ ), a maximum error of $\pm 3 \mathrm{DN}$ (i.e., $\pm 8 \mathrm{nA}$ ) in high gain is expected, with a typical noise of 0.025 DN High Gain peak to peak (i.e, $60 \mathrm{pA}$ ).

\subsubsection{Algorithm verification}

The last test to confirm and validate the full data acquisition and processing chain is the verification of the algorithm performances. This is done by simulating a misaligned S/C configuration [13] and illuminating the SiPMs with the expected irradiance corresponding to this misalignment. This can be done using a simulator that, starting from the modelling of the expected penumbra profile, evaluates the correct LED power required to have the proper output light feeding the SPS as if it was in orbit under the considered configuration. This is done by using the test-bed described in 3.2.1, and the e2e simulation tool described in [13]. The digital output returned by the SPS board are given in input to the metrology algorithm [10] that calculates the corresponding position. By comparing the expected position, that has be set to trigger the input illumination, with the algorithm measurement, the total error budget is estimated.

\section{SOURCE DESCRIPTION AND CALIBRATION}

The LED sources used in the tests have been radiometrically calibrated as a function of the input ADU units with a particular attention to the error budget of the setup, which must be compatible with the accuracy required to the SPS. A critical requirement is that the intensity from the LEDs, given an input current, must remain stable in time, over intervals of the order of hours, to ensure a constant illumination during the SPS calibration. The LED calibration was aimed at studying the response profile as a function of the input DN. As underlined, the complete light source suite is built up of four triplets of LEDs, one triplet for each SPS. A triplet is composed of a low, a medium, and a high intensity LED, with the power of the most intense source roughly one order of magnitude higher than the faintest one. The reason for this comes from the possibility to obtain a combined intensity variation as small as possible to test the required SPS sensitivity to fine changes of illumination, as it will occur when in fight under the solar irradiance, for millimeter order FF lateral misalignment.

The LED intensity is digitally modulated by means of a TTL input with spectral emission centred at $620 \mathrm{~nm}$, with a FWHM of about 23nm. In figure 6, the chromatic response of the spectral profiles of the LEDs, is shown. A drift of the line centroid of $3 \mathrm{~nm}$ is evident within the first 2000 ADU values. We could not measure the
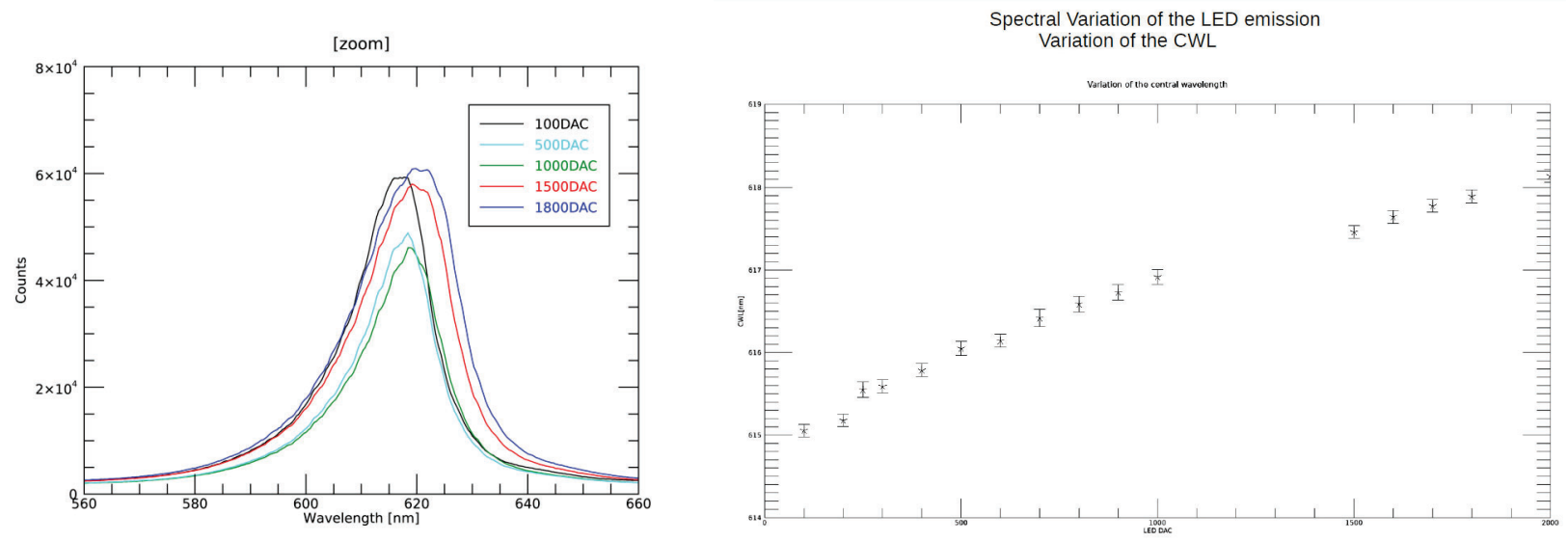

Figure 6. LED emission spectral profiles at different values of input DN, up to 2000 DAC; measurements at higher values were not possible owing to the saturation of the spectrometer detector.

profiles at higher values owing to the saturation of the spectrometer detector, but by extrapolation, we can reasonably confirm that the trend remains within the SPS operative spectral band [500-660] nm. Moreover, we can infer the SPS response calibration in the full range of wavelengths, starting from the results obtained in the 
more restricted emission band of the LEDs. The light from each triplet of LED is collected by three light guides, which are joined into a single optical fibre that is attached to the entrance port of an integrating sphere. The flux is measured by a calibrated photodiode placed close to one of the exit ports of the integrating sphere. The entire system is kept inside a light-tight enclosure, in order to prevent unwanted light reaching the photodiode, as shown in figure 7 . The current from the photodiode is acquired by a picoammeter, capable of detecting the
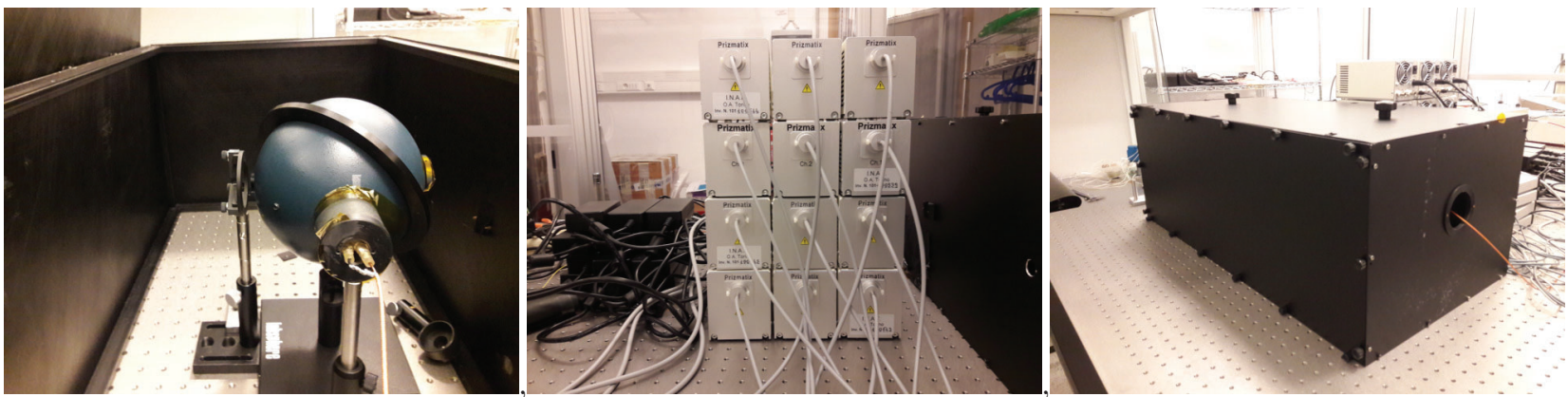

Figure 7. Laboratory set-up.

current variations of the order of $10 \mu \mathrm{A}$ as expected when it is illuminated by the LED light diffused by the integrating sphere. Each measurement is obtained by averaging 50 under-samples, thus obtaining the error from the data dispersion, turning out to be of the order of one thousandth of the value $(0.1 \%)$.

The electronics of the LED controllers has a 12 bits dynamics, corresponding to 4095 different levels of current intensity powering each led: the measured LED emission, corresponding to fixed set of DN values, has been fitted with a polynomial curve. The capacity of the polynomial fit of reproducing the LED emission intensity is of about $5 \%$ that is acceptable for the objective of the SPS calibration. Major efforts have been dedicated to the study of the stability with time, and of the repeatability of the measurements. The conditions of stability were attained after about 30 minutes the system was turned-on (the time interval necessary for the warming up of the system).

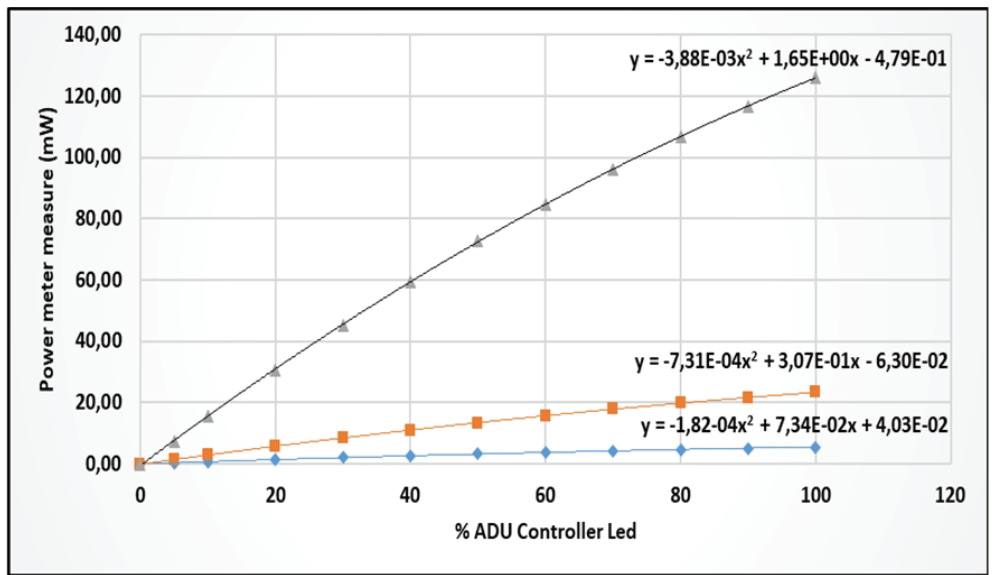

Figure 8. Calibration of the output of a triplet of LEds: polynomial curve fit of the LED intensity as a function of the input DN.

The stability of the LED intensity with time has been tested for each element, at different values of ADU units: an example of the monitoring curve is shown in figure 9. It is evident the presence of the transient lasting about 30 minutes, after that the intensity stabilizes with variations not larger than 0.5 percent. 


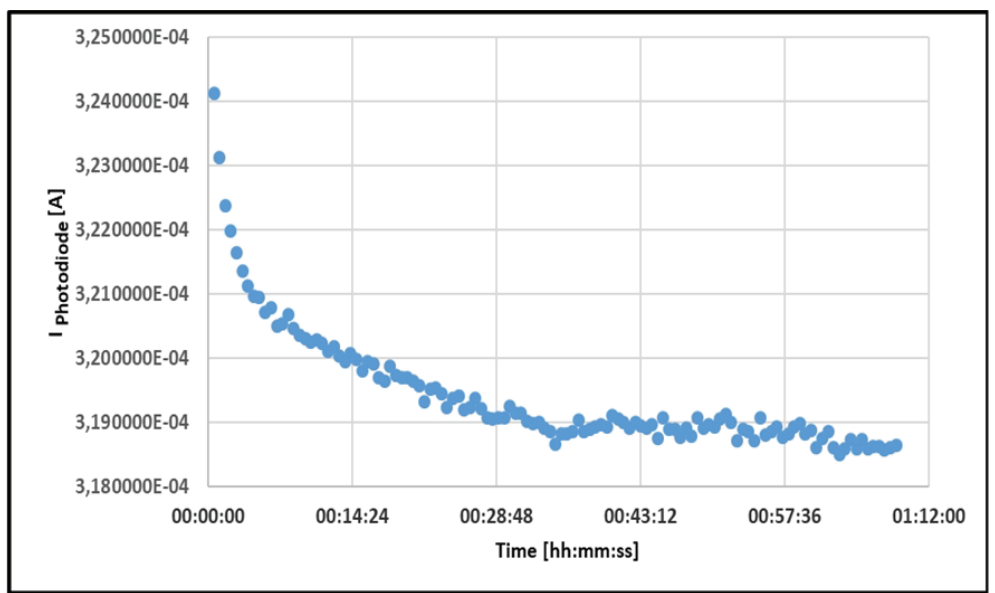

Figure 9. Stability of the LED intensity with time. A warm-up time of about 30 minutes is required.

\section{TEST RESULTS}

The results reported in the next subsections refers to the calibrations on the ADM performed at the INAF Torino, by using the set-up described in section 3.2.

\subsection{Time stability test results}

This test has been performed by illuminating one SiPM at a time with a constant irradiance. We considered three different level of illumination for the HG. Looking at the figure 10, we see that the error increases with the flux,
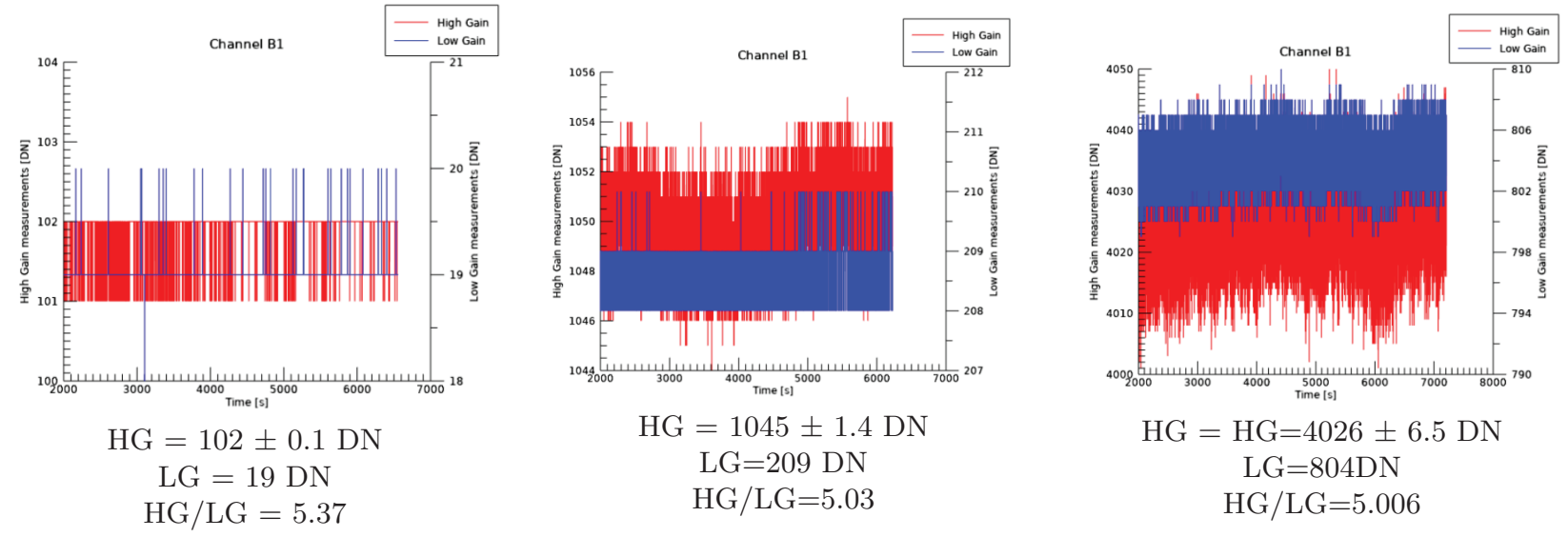

Figure 10. SPS temporal stability at different incident irradiance.

as expected, remaining around $0.1 \%$ of the measured DN (comparable with the source stability), and increasing rapidly while reaching the saturation level. At the nominal alignment conditions, at $\mathrm{ISD}=144 \mathrm{~m}$, we expect to have an SPS readout around $1037 \mathrm{DN}$ so the error expected is about 1.0-1.5DN $(1 \sigma)$. From the qualification of the electronic chain [9], we know that the relation between DN and corresponding lateral shift is $10 \mu \mathrm{m} / \mathrm{DN}$ so the contribution of the temporal stability of the SPS readout, within the requirement box (sec.2), remains of the order of $15 \mu \mathrm{m}$. This contribution is in good agreement with the results obtained by performance analysis and it will be included in the overall error budget to confirm the final error, with the contribution of the electronics and of the numerical calculation (algorithm). 


\subsection{Radiometric calibration results}

In this test we verified how the HG and LG SPS response varies between the tested SiPM under the same illumination. Results are given in figure 11: the correction coefficients $k_{i}$ to be used for the equalization of the readouts are also reported.
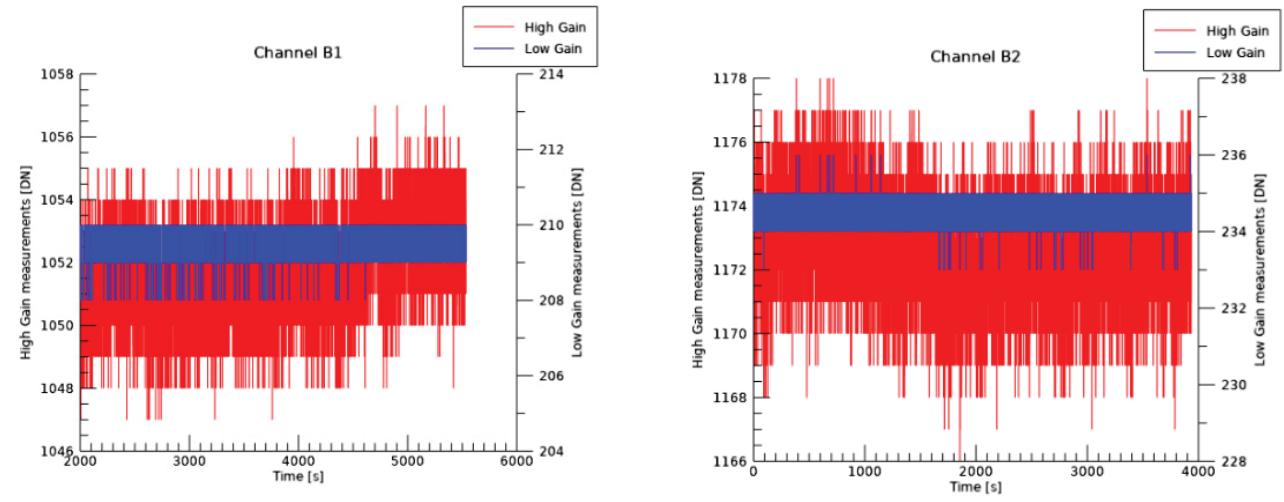

$$
\begin{gathered}
\mathrm{HG}=1052 \mathrm{DN} ; \mathrm{LG}=209 \mathrm{DN} \\
\mathrm{HG} / \mathrm{LG}=5.03 ; k_{i}=0.982
\end{gathered}
$$
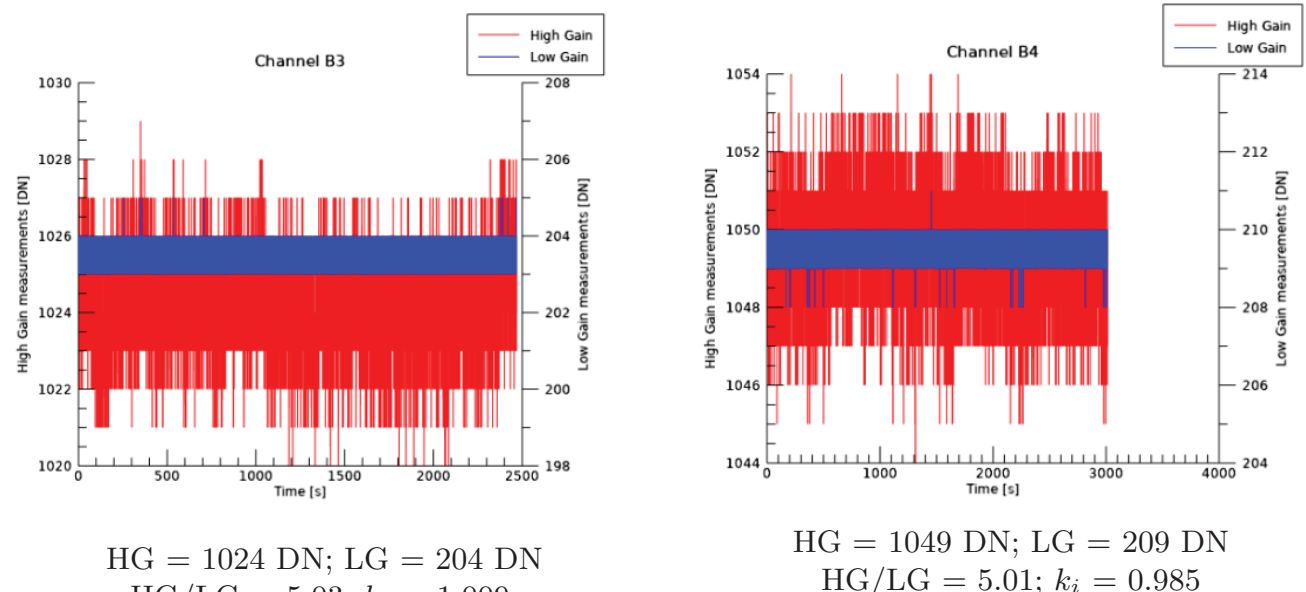

$$
\begin{gathered}
\mathrm{HG}=1024 \mathrm{DN} ; \mathrm{LG}=204 \mathrm{DN} \\
\mathrm{HG} / \mathrm{LG}=5.03 ; k_{i}=1.009 ;
\end{gathered}
$$

$$
\begin{gathered}
\mathrm{HG}=1172 \mathrm{DN} ; \mathrm{LG}=234 \mathrm{DN} \\
\mathrm{HG} / \mathrm{LG}=5.001 ; k_{i}=0.881
\end{gathered}
$$

$\mathrm{HG} / \mathrm{LG}=5.01 ; k_{i}=0.985$

Figure 11. Radiometry calibration results.

This test confirmed what important is the knowledge of the real response of each SPS and the ratio between different SPS readouts in order to provide the metrology algorithm with the best estimation of the $k_{i}$ factors needed to start in-flight operation. The $k_{i}$ factors enter within the so-called "reconfigurable parameters" [10] that, starting from the reference values obtained by the on-ground calibration and validation, will be updated and refined by periodic in-flight calibration for the entire duration of the mission.

\subsection{Spectral dependence of the radiometric calibration results}

The spectral calibration has been done to verify how the corrective factors $k_{i}$ vary with the wavelenght, within the SPS operative waveband. To perform this test, we used a Oriel 66884 QTH source (with flux stabilizer) directly coupled with a monochromator Oriel MS257. On the exit slit of the monochromator, the light is injected into a optical fibre. Before attaching the fiber to the counter-flange to illuminate the ADM, a verification is done using a calibrated spectrograph. In the following figure 12, the measured variation of $k_{i}$ factor is shown. 


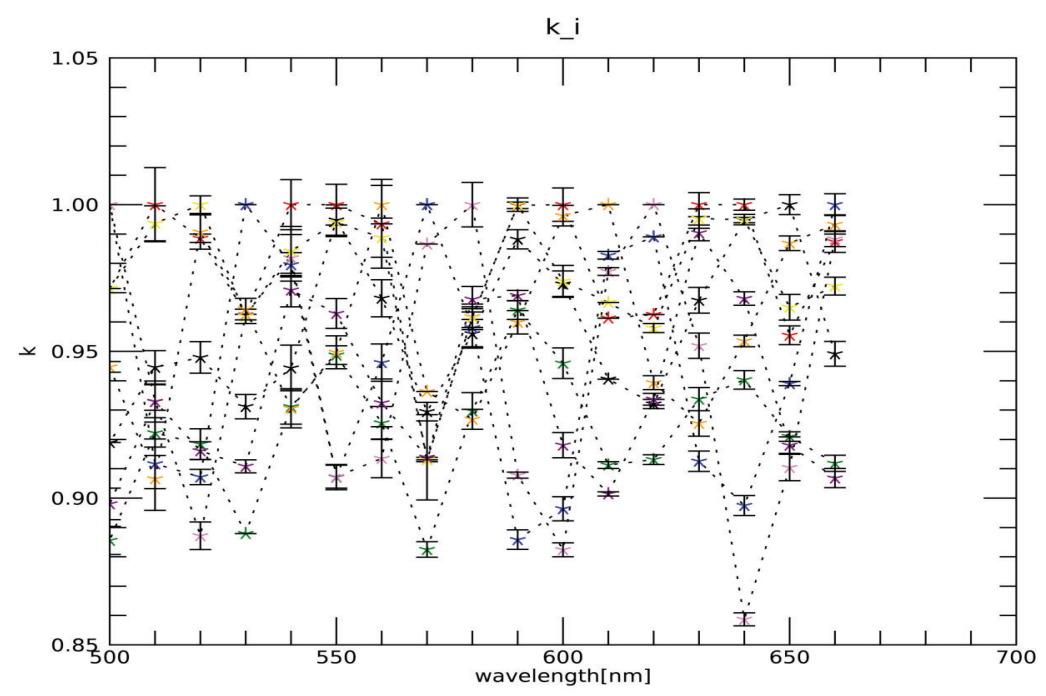

Figure 12. Variation of the $k_{i}$ factors within the SPS waveband.

\section{CONCLUSIONS}

In this paper, we review the laboratory testbed used for the on-ground calibration of the Shadow Position Sensor (SPS) one of the metrology system on board of the PROBA3 mission of the European Space Agency. The PROBA3 mission is a cornerstone mission for the verification and validation of multiple metrology concepts for the realization and maintenance of a two spacecraft formation flying in a high elliptic orbit. The SPS is the metrology system that will provide the most accurate measurement of the relative and absolute alignment of the formation. The tool being used for confirming the correct alignment of the formation is a giant coronagraph, named ASPIICS, with the telescope installed one satellite and the external occulter on the other satellite, placed at the inter-satellite distance of $144 \mathrm{~mm}$. The SPS consists of a system of 8 silicon photomultipliers positioned around the entrance pupil of the ASPIICS telescope aimed at monitoring the symmetry of the penumbra projected by the external occulter. The high accuracy expected on the lateral plane is of the order of $0.5 \mathrm{~mm}$ and satisfying this requirement asks for an accurate characterization and calibration of the SPS system, both on-ground and inflight. The paper provides an insight on the laboratory set-up and on the preliminary results obtained during the on-ground calibration campaign. This campaign is currently in progress by testing the Advanced Demonstration Model of the SPS. In the next future, the calibration will be finalized testing the flight model by adopting the same set up described in this work and providing the system with the complete characterization of the metrology chain of the FM SPS.

\section{ACKNOWLEDGMENTS}

The authors acknowledge the support provided by the PROBA-3 Managerial and Technical Staff of the European Space Agency (ESA) within the contract with the Centre Spatial de Liege (CSL) and subcontractors, subscribed for the Payload Instrument design and development (C/D Phases).

\section{REFERENCES}

[1] Escoubet, C., Schmidt, R., and Goldstein, M., "CLUSTER - Science and Mission Overview," in [Space Science Reviews], 79, 11-32, Kluwer Academic Publishers (1997). https://doi.org/10.1023/A:1004923124586.

[2] Kapilal, V., A.G.Sparks, Buffington, J., and Qiguo, Y., "Spacecraft formation flying: dynamics and control," Proceedings of the 1999 American Control Conference 62, 4137-4141 (1999). doi: 10.1109/ACC.1999.786328.

[3] Bodin, P., Larsson, R., Nilsson, F., and et al., "PRISMA: An In-Orbit Test Bed for Guidance, Navigation and Control Experiments," Journal of Spacecraft and Rockets 46(3), 615-623 (2009). 
[4] Lamy, P., L.Dame, Vives, S., and Zhukov, A., "ASPIICS: a giant coronagraph for the ESA/PROBA-3 Formation Flying Mission," Proc. of SPIE Space Telescopes and Instrumentation: Optical, Infrared, and Millimeter Wave $\mathbf{7 7 3 1}$ (2010). https://doi.org/10.1117/12.858247.

[5] Baccani, C., Landini, F., Romoli, M., and et al., "Preliminary evaluation of the diffraction behind the PROBA 3/ASPIICS optimized occulter," Proc. of SPIE Space Telescopes and Instrumentation: Optical, Infrared, and Millimeter Wave 9904 (2016). doi: 10.1117/12.2232534.

[6] Contreras, R., Penin, L., Marco, V., and et al., "PROBA-3: High Precision Formation Flying in HEO," Advances in the Astronautical Sciences Guidance, Navigation and Control 159 (2017).

[7] Loreggia, D., Fineschi, S., Capobianco, G., Bemporad, A., Cast, M., Landini, F., Nicolini, G., Zangrilli, L., Massone, G., Noce, V., Romoli, M., Terenzi, L., Morgante, G., Belluso, M., Thizy, C., Galy, C., Hermans, A., Franco, P., Pirard, A., Rossi, L., Buckley, S., Spillane, R., O'Shea, M., Galano, D., Versluys, J., Hernan, K., and Accatino, L., "Metrology on-board PROBA-3: The shadow position sensors subsystem," Journal of Advances in Space Research (2020). https://doi.org/10.1016/j.asr.2020.08.004.

[8] Capobianco, G., Fineschi, S., Loreggia, D., and et al., "The in-flight calibration of the shadow position sensors, optical metrology system of the esa/proba-3 formation flying mission," International Workshop on Satellite Constellation and Formation Flying (2019).

[9] Noce, V., Loreggia, D., Capobianco, G., Fineschi, S., Bemporad, A., Casti, M., Buckley, S., Romoli, M., Focardi, M., Belluso, M., Thizy, C., Hermans, A., Galano, D., and Versluys, J., "Metrology onboard PROBA-3: The shadow position sensors subsystem," Journal of Advances in Space Research (2020). https://doi.org/10.1016/j.asr.2020.08.004.

[10] Casti, M., Bemporad, A., Fineschi, S., Capobianco, G., Loreggia, D., Noce, V., Landini, F., Thizy, C., Galano, D., and Rougeot, R., "PROBA-3 formation-flying metrology: algorithms for the shadow position sensor system," in [International Conference on Space Optics - ICSO 2018], Proc. SPIE 11180, 1118082 (2019). https://doi.org/10.1117/12.2536209.

[11] Bemporad, A., Baccani, C., Capobianco, G., Fineschi, S., Focardi, M., Landini, F., Loreggia, D., Massone, G., Nicolini, G., Noce, V., Pancrazzi, M., Romoli, M., Buckley, S., O'Neill, K., Renotte, E., Servaye, J. S., and Thizy, C., "The Shadow Positioning Sensors (SPS) for formation flying metrology on-board the ESAPROBA3 mission," in [Solar Physics and Space Weather Instrumentation VI], Proc. SPIE 9604, 96040C (2015). https://doi.org/10.1117/12.2191829.

[12] Capobianco, G., Loreggia, D., Fineschi, S., Focardi, M., Bemporad, A., Casti, M., Noce, V., Landini, F., Baccani, C., Pancrazzi, M., Romoli, M., Massone, G., Nicolini, G., Buckley, S., O’Neill, K., Cernica, I., Purica, M., Badianu, E., Thizy, C., Servaye, J.-S., Mechmech, I., and Renotte, E., "The satellite formation flying in lab: PROBA-3/ASPIICS metrology subsystems test-bed," in [Space Telescopes and Instrumentation 2016: Optical, Infrared, and Millimeter Wave], Proc. SPIE 9904, 99046E (2016). https://doi.org/10.1117/12.2233207.

[13] Capobianco, G., Amadori, F., Fineschi, S., Bemporad, A., Casti, M., Loreggia, D., Noce, V., Pancrazzi, M., Landini, F., Thizy, C., Rougeot, R., Galano, D., and Versluys, J., "Formation Flying performances simulator for the Shadow Position Sensors of the ESA PROBA-3 mission," in [International Conference on Space Optics - ICSO 2020 (this conference)], (2021). 